\title{
Article \\ Microfluidic Platform combined with a Dark Field Imaging System for Quantification of E. coli Contamination in Water
}

\author{
Anna Malec ${ }^{1}$, Christoph Haiden ${ }^{1,2}$ Georgios Kokkinis, ${ }^{1,3}$ and Ioanna Giouroudi 1,4,5* \\ 1 Institute of Sensors and Actuators, Faculty of Electrical Engineering and Information Technology, Tech- \\ nische Universität Wien, Vienna, Austria; annmalec@gmail.com \\ 2 ZKW Elektronik GmbH, Austria; Christoph.Haiden@zkw-elektronik.at \\ 3 Silicon Austria Labs, Villach, Austria; giorgos.kokkinis@gmail.com \\ 4 BioSense Institute, Novi Sad Serbia; \\ 5 TUW Doctoral School, Technische Universität Wien, Vienna, Austria; ioanna.giouroudi@tuwien.ac.at \\ * Correspondence: ioanna.giouroudi@tuwien.ac.at; Tel.: (optional; +43158801405601)
}

\begin{abstract}
In this paper, we present a method for detecting and quantifying pathogens in water samples. The method proposes a portable dark field imaging and analysis system for quantifying E. coli concentrations in water after being labeled with magnetic particles. The system utilizes the tracking of moving micro/nano objects close to or below the optical resolution limit confined in small sample volumes $(\sim 10 \mu \mathrm{l})$. In particular, the system analyzes the effect of volumetric changes due to bacteria conjugation to magnetic microparticles (MP) on their Brownian motion while being suspended in liquid buffer solution. The method allows for a simple inexpensive implementation and the possibility to be used as point-of-need testing system. Indeed, a working prototype is demonstrated with the capacity of quantifying $E$. coli colony forming units (CFU) at a range of $1 \times 10^{3}-6 \times 10^{3} \mathrm{CFU} / \mathrm{mL}$.
\end{abstract}

Keywords: pathogen detection, bacteria quantification, dark field imaging, hydrodynamic diameter, E.coli, biosensing, water contamination, magnetic microparticles

\section{Introduction}

Microbial contamination of water supplies can lead to serious illness and even death of livestock and human populations, therefore requiring pathogen detection for water quality control. Rapid and multiplexed measurement of such water-borne pathogens is vital and the challenge is to instantly detect different types of pathogens in these liquid samples, on the spot and in real-time. Several principles are available for bacteria detection, including traditional culturing, enzyme-linked immunosorbent assay methods (ELISA), immunomagnetic separation with fluorescence staining, fluorescent bacteriophage assay combined with flow cytometry, solid phase fluorescent capillary immunoassay and time resolved fluorescence immunoassay [1-3]. More recent approaches include polymerase chain reaction (PCR), evanescent wave fibre optic biosensors, PCR-acoustic wave sensor combinations, surface plasmon resonance (SPR) sensors, interferometric array sensors, electrochemical impedance spectroscopy-based sensors, giant magnetoresistance (GMR) sensors and MEMS sensors, e.g., functionalized cantilever sensors, such as piezoelectric-excited millimeter-sized cantilevers (PEMC) [4-6]. However, conventional water monitoring techniques are typically based on laboratory analyses of representative field-collected samples, implying considerable effort and expense for sample collection and transport as well as the risk of sample change before analysis can take place [7-9]. Usually, this kind of analysis equipment is not portable and furthermore, the current state of the art often fails to address measurements in a timely manner combined with multiple detection analyses and the ability to quantify the contaminants concentration in highly diluted water samples [10-11]. Obviously, there is an increasing demand of robust and efficient techniques of contaminant detection and of monitoring devices to save analysis time, reagents, and amount of sample, as well as the development of portable systems. 
E.coli is a gram-negative, commonly present in nature, rod-shaped bacteria of approximately $0.5 \mu \mathrm{m}$ width and $2 \mu \mathrm{m}$ length [12]. Due to its small size, optical microscopy studies regarding its quantitative dynamics over a life cycle or in response to modifications of external/internal conditions are limited by optical resolution and low contrast of biological matter. In previous work, we described velocity tracking of magnetically labelled bacteria under magnetophoretic actuation using fluorescence microscopy [13] and magnetic stray field detection [14], as well as dark field microscopy and tracking of magnetic sub-micron particles for size measurements [15]. Here, we present a quick and inexpensive approach for E.coli detection and quantification based on dark field video microscopy and particle tracking, allowing simple and rapid analysis of multiple individual micromarker particles conjugated with E.coli. The main goal of the current study was to analyze the effect of volumetric changes due to bacteria conjugation to magnetic microparticles (MP) on their Brownian motion while being suspended in liquid buffer solution. Also, the effect of different concentrations on the binding to the microparticles and resulting hydrodynamic diameter increase of loaded magnetic particles (LMP) were evaluated. The system's design is based on criteria such as portability, sensitivity, sample handling, cost-efficiency, and biocompatibility. It is easy to implement and could be operated without the need for highly qualified personnel or sophisticated laboratory environment.

\section{Materials and Methods}

\subsection{Working Principle}

Particles suspended in liquid will collide with fast-moving fluid molecules and change their trajectories in uncoordinated, contingent step-manner (Brownian motion). The extent of a particle's Brownian motion is characterized by a diffusion coefficient $D$, which is dependent not only on a particle diameter (i.e. output variable of interest) but also on other variables like temperature and sample viscosity [16]. This relation is described by the Stokes-Einstein equation $d=k_{B} T / 3 \pi \eta D$, where $d$ is the hydrodynamic particle diameter, $D$ is the diffusion coefficient of a spherical particle, $k_{\mathrm{B}}$ is the Boltzmann constant, $T$ is the absolute temperature, and $\eta$ is the solvent viscosity.

A schematic representation of the trajectory of Brownian motion of an individual particle together with a graphical explanation of mean square displacement (MSD) can be seen in Figure 1.

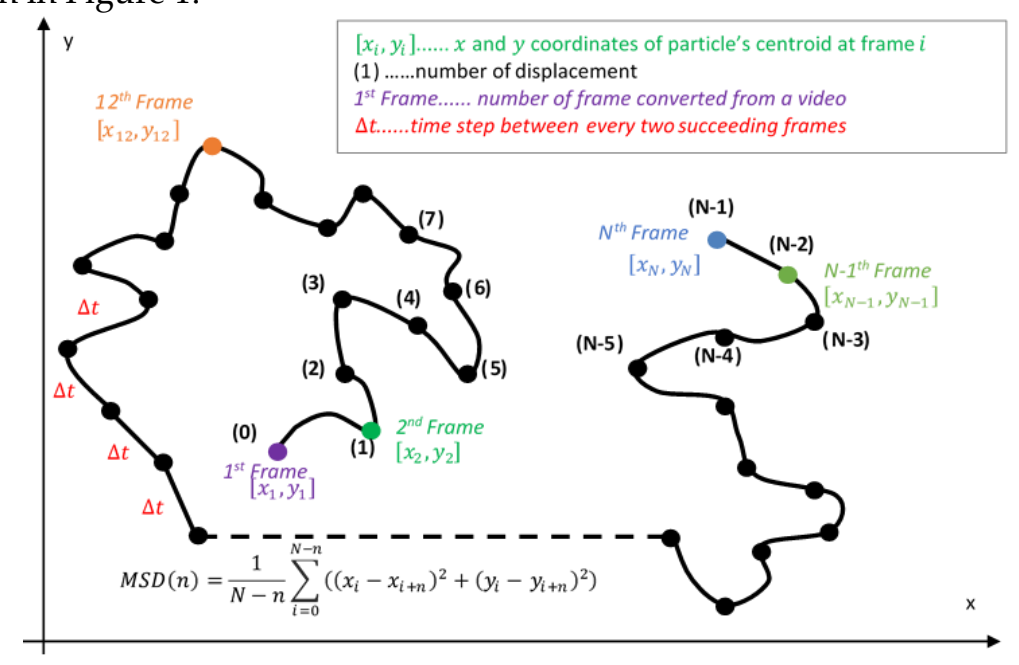

Figure 1. Principle of an MSD calculation from a schematic representation of the 2D trajectory of an individual particle undergoing Brownian motion. Time interval $\Delta t$ between each succeeding frame $i=1, \ldots N$ stays unchanged, while $x_{\mathrm{i}}$ and $y_{\mathrm{i}}$ coordinates correspond to a variable position of the particle's centroid captured at a specific frame $i$. 
It is possible to track these tiny particle motions due to a combination of light scattering, i.e., dark field illumination to visualize small particles, and an appropriate particle tracking system [17]. To follow the dynamic processes and interactions within a liquid sample, the subsequent frames from the camera's recordings are collected and analyzed. Trajectories of multiple particles can be tracked simultaneously in one field of view (FOV) over a desired time period. Provided that the particles are suspended in a static fluid where viscosity and temperature do not change over time and for all sets of measurements, the diameters of the particles suspended in a fluid can be determined and compared for each sample set.

Binding of species such as microorganisms to surface functionalized particles will effectively change the overall particle size, which translates into an increase of their hydrodynamic diameter (see Figure 2). Larger particles will collide more likely and more often with the surrounding buffer molecules whereas smaller particles will move more freely within the liquid. This results in a decreased diffusion coefficient $D$ and hence decreased MSD for larger, loaded particles compared to smaller, unloaded ones.

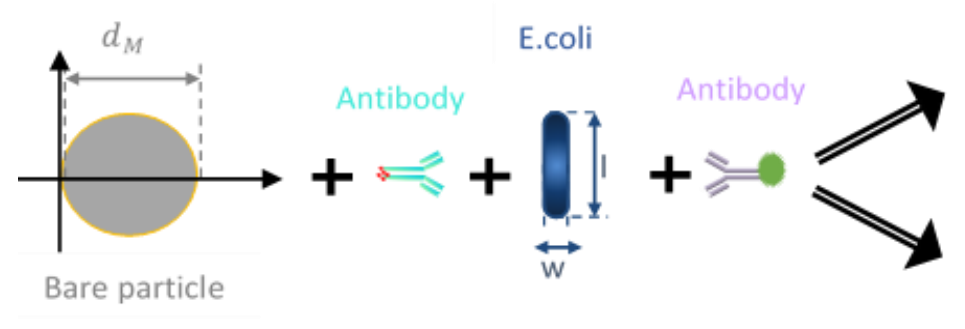

ure

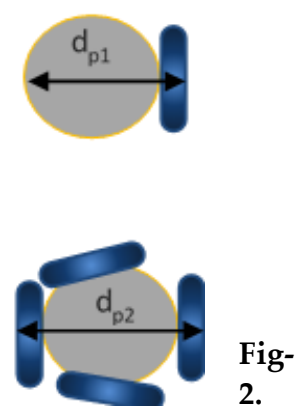

Schematic representation of E.coli attachments and volumetric changes of a particle. The number of attachments depends on the E.coli bacteria concentration within the buffer solution.

A conventional microscopy and particle tracking setup would comprise an upright microscope and camera system to observe and track 2D-diffusion of particles suspended in a transparent liquid sample cell, which is in the simplest case composed of liquid sample sandwiched between two glass slides. While this would work well for smaller and particularly lighter particles [17], larger particles, such as the micromarkers used in this work, would inevitably sediment to the bottom of the glass sample chamber and cause unwanted attachments of biological agents (i.e., attachment of E.coli/antibodies from the microparticle-E.coli complex) to the glass slide.

To avoid this case, the biological liquid containing pathogen-loaded-micromarkers is entrapped in a sample cell which is positioned on the vertical stage of a custom microscope setup and viewed with a microscope system perpendicular to the plane of the sample (Figure 3) $[18,19]$. Due to this arrangement, Brownian motion in $x$ - $y$-direction is superimposed by gravitational movements along the vertical $y$-axis (with the $z$-axis being the horizontal axis, i.e., the light path). This results in a sedimentation movement along the $y$-axis that would distort Brownian motion along this direction. Therefore, in order to calculate the particle diameter from 2D particle tracking data, $y$-components of the MSD are excluded and only the horizontal $x$-component is considered [18]. Thus, for displacements along a single dimension, the relation is $\operatorname{MSD}_{\times}(n)=2 D n \Delta t$ with $n=1 \ldots N-1, N$ the total number of recorded displacements of the particle trajectory and $\Delta t$ the time interval between two consecutive frames. Fitting of the first two points of the MSDx(n) curve is done to obtain the diffusion coefficient $[20,21]$, and consequently calculate the hydrodynamic diameter of the particle from the Stokes-Einstein equation. 


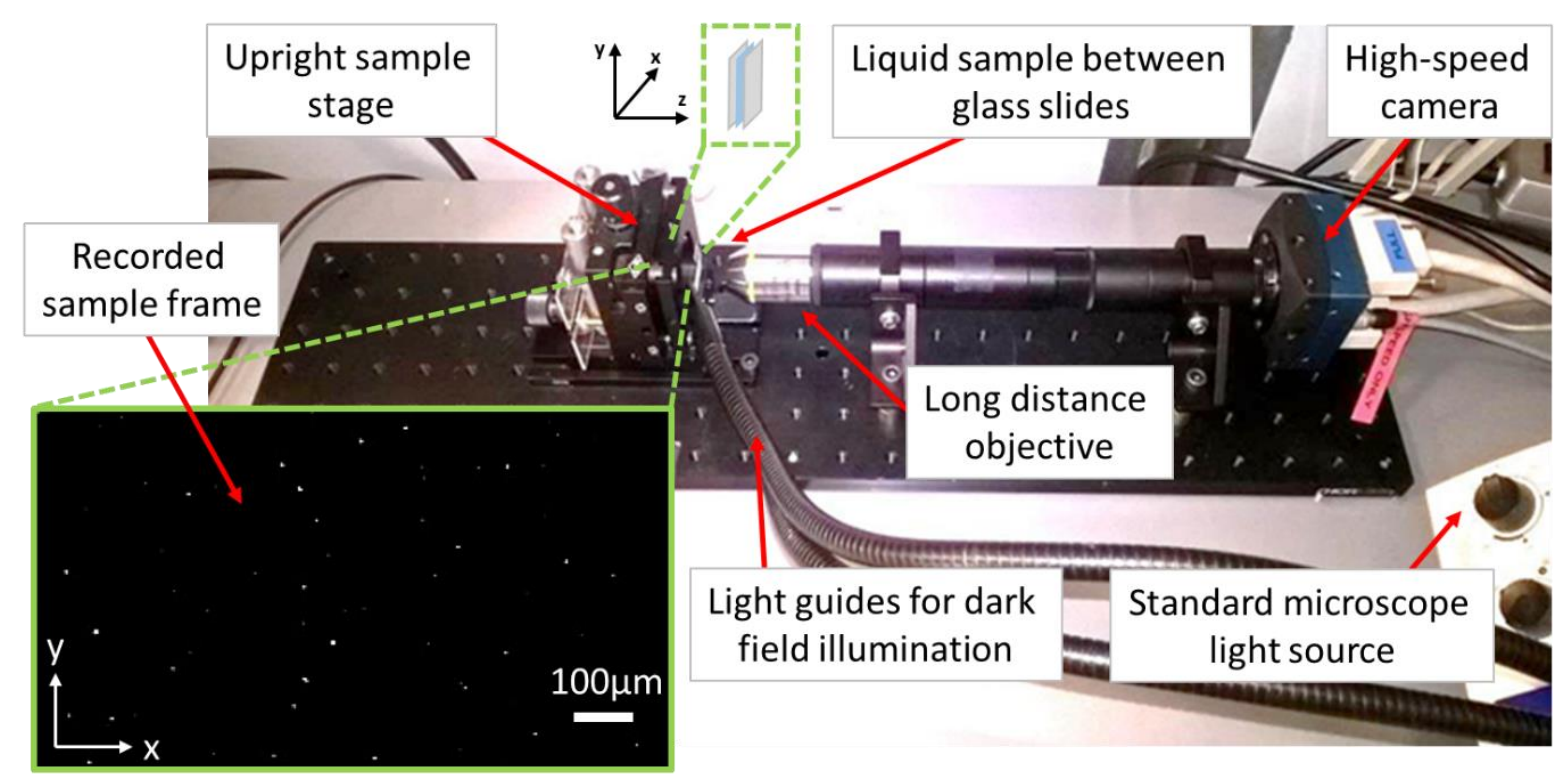

Figure 3. Experimental setup for combined dark field microscopy and Brownian quantification method equipped with a vertical stage, sample holder and light source located on the same side as the objective and mounted camera. Inset: an individual output frame image of a selected field of view.

\subsection{Optical system}

Dark field illumination allows imaging of particles below the optical resolution limit $(\sim 1 \mu \mathrm{m}$ with the current optical system) and of larger microparticles alike. Here, a straightforward arrangement for dark-field microscopy was employed: The sample stage was illuminated at such an angle that the scattered light was detected by a camera, while the reflected light could not enter the optical path (see Figure 3). As seen from the inset of Figure 3, an image of bright spots over a dark background corresponding to micromarkers scattering the incoming light was produced. The illumination system was positioned at the side of lens (epi-illumination) and accustomed in a manner to avoid unwanted saturation of camera pixels (saturated pixels cannot provide direct information about incident irradiance [22]). The long working distance (WD) objective with numerical aperture $\mathrm{NA}=0.3$ enabled broad focal depth, so that particles were visible for extended time periods [17]. Therefore, a continuous observation of almost all the particles within a selected FOV was possible. Additionally, the long WD allowed comfortable mounting of the sample cell on the stage and no additional coarse focusing was required if the same magnification arrangements were selected. In order to reduce background scattering noise, smooth surfaces are required. These conditions were sufficiently fulfilled by applying a standard microscopy cover glass slip on the top of the sample.

The laboratory-scale measurement system basically consists of a long WD microscope objective (10x/0.3, Nikon) and a camera (MIKROTRON EoSens MC 1362) mounted with optomechanical components (Thorlabs). The light source was a conventional halogen microscope light source (Zeiss KL 1500 LCD 3000K) with swan neck light guides to illuminate the sample at a shallow angle. Camera software (MotionBLITZDirector2 LTR) was used to record successive video frames. The image resolution of the optical system was determined as $0.88 \mu \mathrm{m} / \mathrm{px}$ with a 1951 USAF Negative Resolution Test Target $3 " \times 3 "$ [17]. For particle tracking purposes, the camera's frame rate and image exposure time needs to be selected in a way to capture differences in scattering between consecutive frames without disruptive motion blurring, however, due to the large particle diameters and hence small diffusivities, the exposure time was set as approximately the time between two consecutive frames, which was $40 \mathrm{~ms}$ at a frame rate of $25 \mathrm{fps}$. 


\subsection{Particle Tracking Principle}

A MATLAB script based on the 2D particle tracking Crocker-Grier algorithm [23] is used to detect the exact position (centroids) of bright, spot-like objects on a dark background appearing over subsequent frames and link these positions to form individual particle trajectories. Details on the tracking method have been described in [13, 17, 18]. Briefly, each frame is bandpass filtered to reduce pixel noise and long range image brightness variations. Then, coarse determination of intensity peaks of multiple spots with several pixels in diameter with pixel accuracy is done for each frame, followed by precise determination of the centroid, resulting in particle positions with sub-pixel accuracy. Once particles are detected and their positions are known for an entire sequence of video images, their locations are matched with successive and preceding frames, i.e., it is determined which particle in a given frame most likely corresponds to the particle in the preceding frame. Due to the relatively low concentration of spots in an image frame, incorrect crosslinking between different particles can be avoided, as elucidated in [17]. Thus, by linking of each detected particle position from all processed frames based on the estimated displacement of a particle between two subsequent frames, complete particle trajectories are generated. That way, all $x$-/y-coordinates of all recorded particles per FOV over time are obtained and can be further processed to calculate the respective diameters, as described above.

\subsection{Sample preparation}

In this research work, the biological target (wild type K-12 E.coli strain) was attached to magnetic microparticles (MP) coated with streptavidin (Dynabeads ${ }^{\mathrm{TM}} \mathrm{M}-280$, ThermoFisher) using biotinylated rabbit polyclonal antibody anti-E.coli (Abcam@ ab20640). The biotin-streptavidin lock-and-key coupling system causes strong noncovalent binding between the streptavidin layer of the particles and the biotin molecules attached on the surface of the antibody and is commonly used to achieve binding of biological species. The microparticles consist of iron nanoparticles in a polymer matrix and have a diameter of $2.8 \mu \mathrm{m}$.

E.coli concentrations ranging from $1 \times 10^{3}$ to $6 \times 10^{3} \mathrm{CFU} / \mathrm{ml}$ were prepared and mixed with washed streptavidin-coated magnetic microparticles. Magnetic separation of E.coliloaded microparticles from the mixing solution in the vial was required in order to resuspend them in fresh PBS-BSA buffer solution. Details on the E.coli binding protocol are stated in [13].

Buffer solution with unloaded or E.coli-loaded particles was pipetted onto a glass slide (10 $\mu$ l drop) and covered with a second glass slide to form an approximately $20 \mu \mathrm{m}$ thick liquid film; the edges were sealed in order to avoid drift, evaporation and movement of glass slides when applied on the vertical sample stage holder.

\section{Results}

Analysis of the samples by means of fluorescence microscopy (Nikon oil 40×) showed the quality of the binding protocol. Numerous loaded magnetic particles were formed for all of the samples with E. coli concentration $\mathrm{c}_{1}=1 \times 10^{3} \mathrm{CFU} / \mathrm{ml}, \mathrm{C}_{2}=2 \times 10^{3} \mathrm{CFU} / \mathrm{ml}$, $\mathrm{C}_{4}=4 \times 10^{3} \mathrm{CFU} / \mathrm{ml}, \mathrm{C}_{6}=6 \times 10^{3} \mathrm{CFU} / \mathrm{ml}$. Figure 4 shows a visible increase in attachments per single MP with increasing concentration of $E$. coli in the liquid sample. For bigger concentrations, the attachments consist of multiple E. coli rods that are bound together (due to antibodies) creating tight visible clods. The arrangement of the attachments for bigger E. coli concentrations is arbitrary, while for smaller concentrations the preferred arrangement is along the long axis of the bacteria. The estimated ratio of the MP's volume to the amount of the biological component declines with increasing concentration. It is apparent from Fig. 4 that with increasing concentration of E. coli, there is also an increase in attachments and therefore a volumetric change of the MP-E. coli compounds, which shall be determined by particle tracking measurements. 


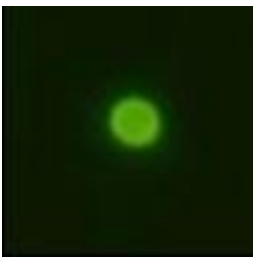

(a)

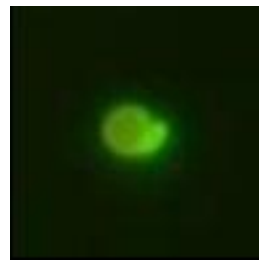

(b)

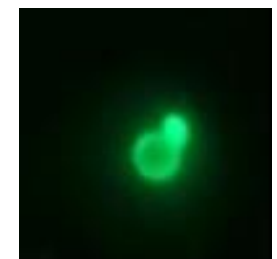

(c)

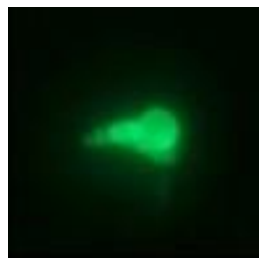

(d)

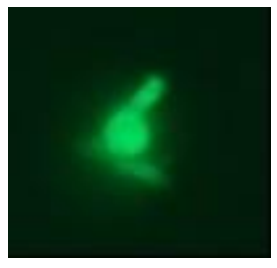

(e)

Figure 4. Fluorescence microscopy image for particles from samples with different E.coli concentration (a) $\mathrm{c}_{0}=0$; $\mathrm{CFU} / \mathrm{ml}$; (b) $\mathrm{c}_{1}=1 \times 10^{3} \mathrm{CFU} / \mathrm{ml}$; (c) $\mathrm{c}_{2}=2 \times 10^{3} \mathrm{CFU} / \mathrm{ml}$; (d) $\mathrm{c}_{4}=4 \times 10^{3} \mathrm{CFU} / \mathrm{ml}$; (e) $\mathrm{C}_{6}=6 \times 10^{3} \mathrm{CFU} / \mathrm{ml}$.

The first set of measurements examines the diameters of bare, reference MPs (without E.coli loadings). The tracking results are represented in Figure 5 in the form of a normalized histogram, where the height of each bar is equal to the probability of selecting an observation within that bin interval), while the $x$-axis correspond to the hydrodynamic diameter calculated from Brownian motion measurements. The normalization was introduced in order to be able to compare distributions of all samples with different amounts of detected particles (i.e. different amount of observations).

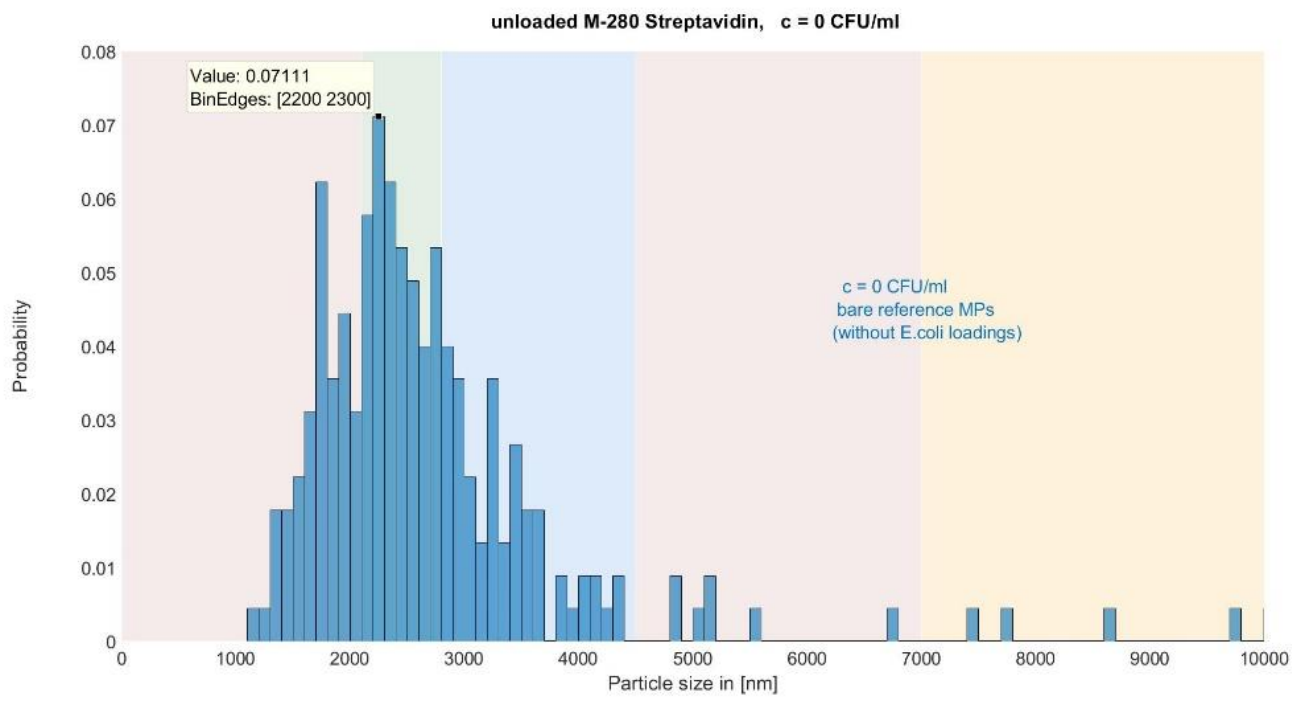

(b)

Figure 5. Hydrodynamic diameter distribution determined by tracking of bare MPs (E. coli concentration $\left.\mathrm{c}_{0}=0 \mathrm{CFU} / \mathrm{ml}\right)$.

The highest peak interval for a sample with bare magnetic particles M-280 Streptavidin (without the layer of biotinylated antibodies) ranges between $2200 \mathrm{~nm}$ and $2300 \mathrm{~nm}$ [see Figure 5]. The values above $10000 \mathrm{~nm}$ are outliers resulting from impurities (e.g., dust) and are not included in further analysis. Based on this reference distribution and assuming that the change of diameter corresponds to the size of E.coli attached, the following coarse regions are defined and estimated for the analysis of the next samples, where the biological component is already present (considering an overlap between the regions due to each species' distribution width) :

- $\quad 0 \mathrm{~nm}<\mathrm{d}<2100 \mathrm{~nm}$ - unattached E.coli suspended in the buffer solution

- $\quad 2100 \mathrm{~nm}<\mathrm{d}<2800 \mathrm{~nm}$ - bare MPs (without loadings)

- $\quad 2800 \mathrm{~nm}<\mathrm{d}<4500 \mathrm{~nm}$ - LMP (single E.coli loading as E.coli width is $\sim 0,5 \mu \mathrm{m}$ and length $\sim 2 \mu \mathrm{m}$

- $\quad 4500 \mathrm{~nm}<\mathrm{d}<7000 \mathrm{~nm}$ - LMP (multiple E.coli loadings)

- $\quad 7000 \mathrm{~nm}<\mathrm{d}<10000 \mathrm{~nm}$ - agglomerations of multiple LMP and/or E.coli 
- $\quad \mathrm{d}>10000 \mathrm{~nm}$ - impurities (dust), outliers

It is assumed that the maximal size of the single LMP (i.e., MP loaded with single E.coli rod) equals to $2.8 \mu \mathrm{m}+0.5 \mu \mathrm{m}=3.3 \mu \mathrm{m}$ or $2.8 \mu \mathrm{m}+2 \mu \mathrm{m}=4.8 \mu \mathrm{m}$ depending on the binding's arrangement. Since the highest peak on Figure 5 is shifted to the left (bare MP size is smaller than supplier specs), so are the other regions.

Figure 6 represents the tracking results for liquid where E.coli of concentration $\mathrm{c}=10^{3} \mathrm{CFU} / \mathrm{ml}$ are already present.

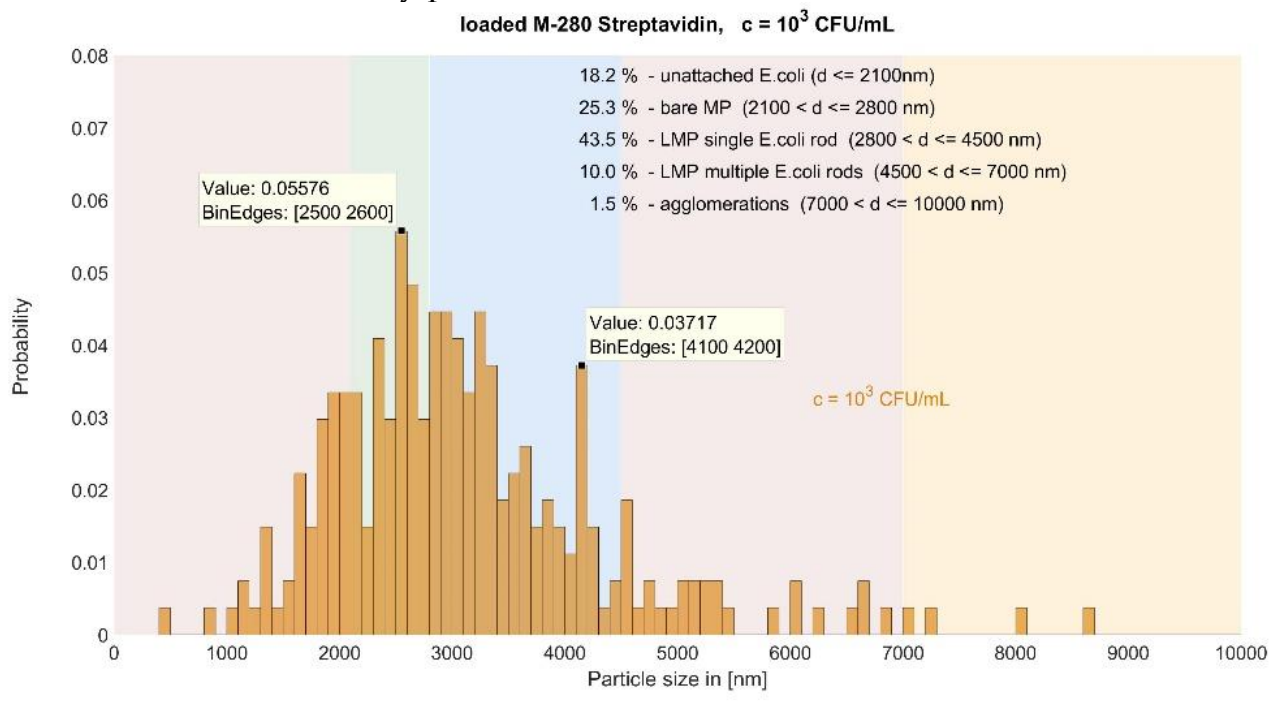

Figure 6. Distributions for the calculated hydrodynamic diameter for E.coli concentration $\mathrm{C}_{1}=10^{3} \mathrm{CFU} / \mathrm{ml}$.

The distribution of the particle-E.coli compound is shifted to the right in comparison to bare MPs. The values of the calculated hydrodynamic diameter are therefore greater than for the reference sample. It is observed that the distributions for other concentrations $\left(\mathrm{c}_{2}=2 \times 10^{3} \mathrm{CFU} / \mathrm{ml}\right.$ vs $\mathrm{c}_{4}=4 \times 10^{3} \mathrm{CFU} / \mathrm{ml}$ vs $\mathrm{c}_{6}=6 \times 10^{3} \mathrm{CFU} / \mathrm{ml}$ ) represented on Figure $7-9$ are also changing in accordance with E.coli concentration.

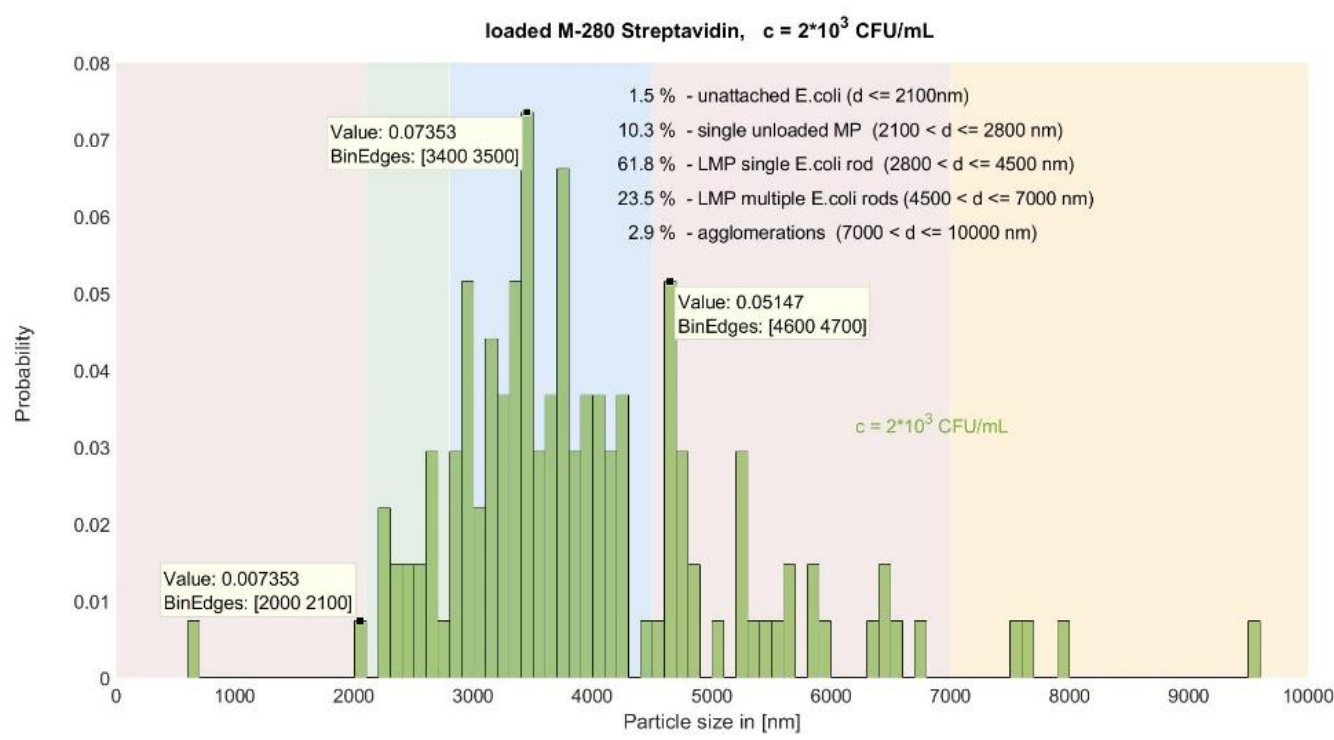

Figure 7. Distributions for the calculated hydrodynamic diameter for E.coli concentration $\mathrm{C}_{2}=2 \times 10^{3} \mathrm{CFU} / \mathrm{ml}$. 


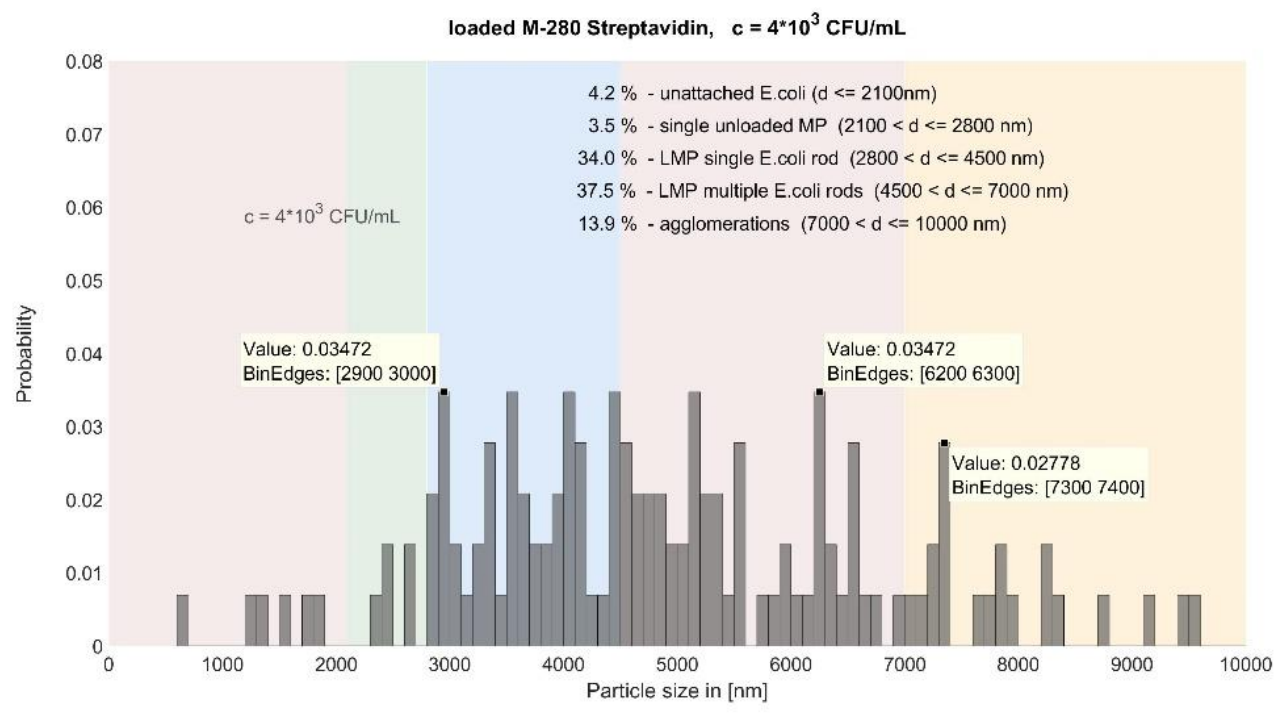

Figure 8. Distributions for the calculated hydrodynamic diameter for E.coli concentration $\mathrm{C}_{4}=4 \times 10^{3} \mathrm{CFU} / \mathrm{ml}$.

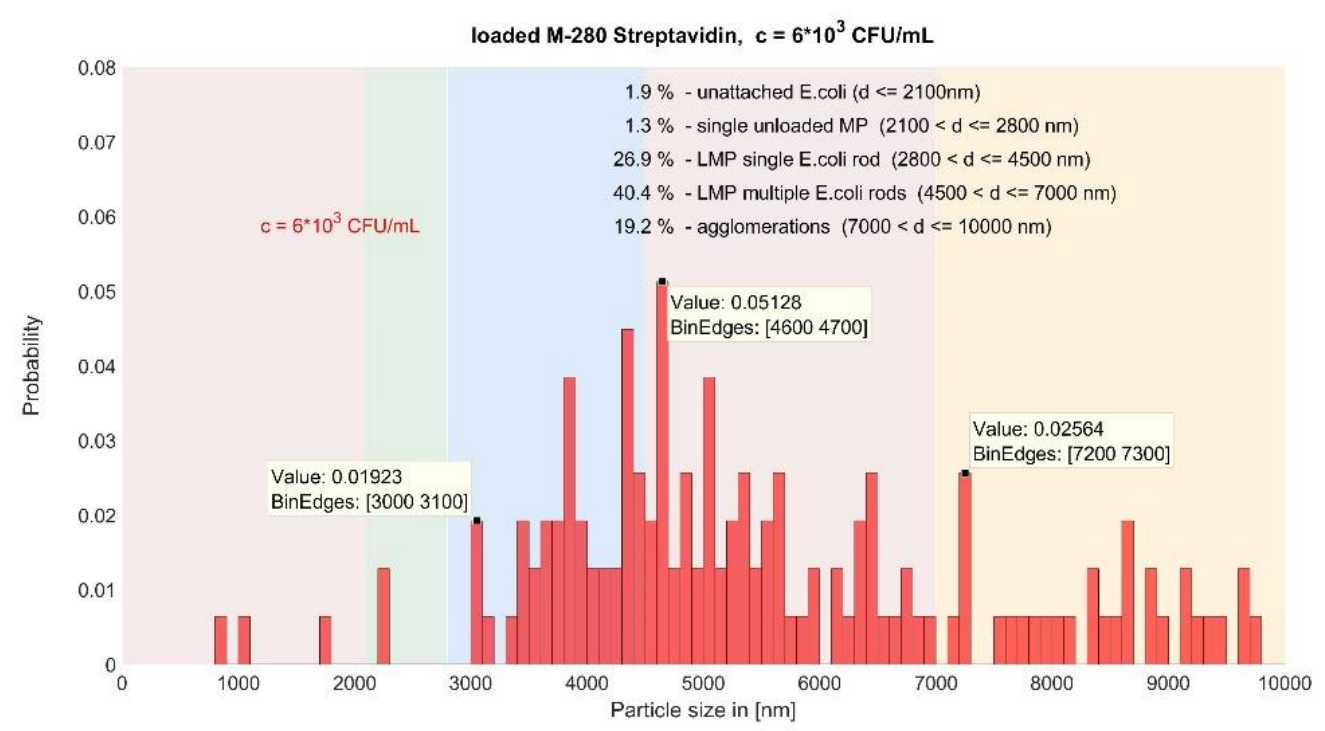

Figure 9. Distributions for the calculated hydrodynamic diameter for E.coli concentration $\mathrm{C}_{6}=6 \times 10^{3} \mathrm{CFU} / \mathrm{ml}$.

Through the analysis of the histograms, it is possible to evaluate which distribution would correspond to E.coli concentration $\mathrm{c}_{0}$ and which one to $\mathrm{c}_{2}, \mathrm{c}_{4}$ or $\mathrm{c}_{6}$. It is proved that the calculated hydrodynamic diameter is related to the E.coli concentration (the bigger the concentration is, the bigger the radius gets). To compare/summarize all of the samples, a non-parametric kernel smoothing function was estimated to find a fit for the histograms [17]. The findings, presented in Figure 10, confirm again the association between calculated hydrodynamic diameter and bacteria concentration. The peak values for the samples: $\quad \mathrm{c}_{0}=0 \times 10^{3} \mathrm{CFU} / \mathrm{ml}, \quad \mathrm{c}_{1}=1 \times 10^{3} \mathrm{CFU} / \mathrm{ml}, \quad \mathrm{c}_{2}=2 \times 10^{3} \mathrm{CFU} / \mathrm{ml}, \quad \mathrm{c}_{4}=4 \times 10^{3} \mathrm{CFU} / \mathrm{ml}$, $\mathrm{C}_{6}=6 \times 10^{3} \mathrm{CFU} / \mathrm{ml}$ ascend in the following manner: $2391 \mathrm{~nm}, 2830 \mathrm{~nm}, 3498 \mathrm{~nm}, 4172 \mathrm{~nm}$, $4567 \mathrm{~nm}$ respectively. 


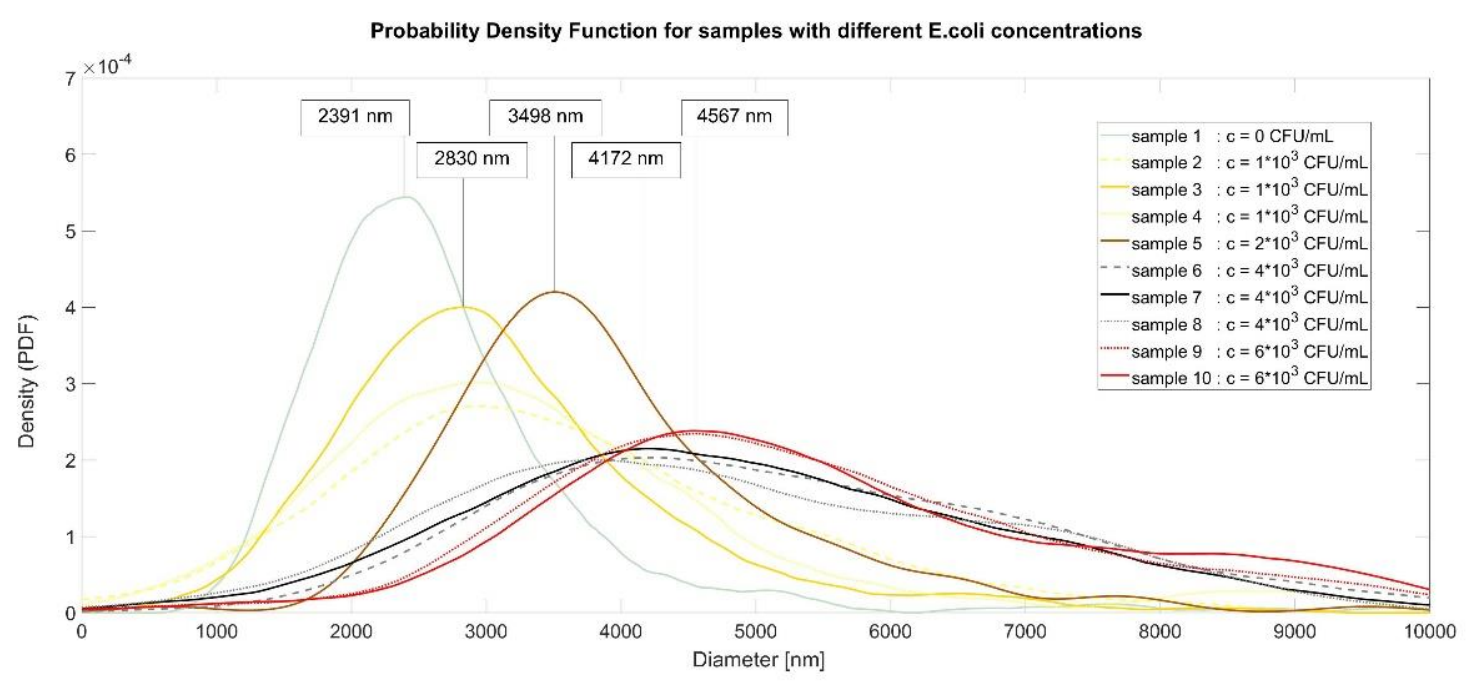

Figure 10. Quantification of E. coli content by comparing peak values of hydrodynamic diameter distributions. This figure represents the fitting curves for samples with various $E$. coli concentrations $\left(\mathrm{c}_{0}=0 \times 10^{3} \mathrm{CFU} / \mathrm{ml}, \mathrm{c}_{1}=1 \times 10^{3} \mathrm{CFU} / \mathrm{ml}, \mathrm{c}_{2}=2 \times 10^{3} \mathrm{CFU} / \mathrm{ml}, \mathrm{C}_{4}=4 \times 10^{3} \mathrm{CFU} / \mathrm{ml}, \mathrm{C}_{6}=6 \times 10^{3} \mathrm{CFU} / \mathrm{ml}\right)$. There is an obvious trend towards an increase in estimated particle diameter with greater $E$. coli concentration. To all samples, the same fitting parameters were applied.

\section{Discussion}

The comparison of all size distributions presented in Figure 10 confirms the association between measured hydrodynamic diameter and bacteria concentration, with peak values increasing from $2.39 \mu \mathrm{m}\left(\mathrm{c}_{0}=0 \times 10^{3} \mathrm{CFU} / \mathrm{ml}\right)$ up to $4.57 \mu \mathrm{m}\left(\mathrm{c}_{6}=6 \times 10^{3} \mathrm{CFU} / \mathrm{ml}\right)$. The difference in the measured hydrodynamic diameter between co and c1 samples amounts to approximately $0.4 \mu \mathrm{m}$, which is already close to the width of a single E. coli. According to the equivalent diameter model detailed in [24], an elongated spheroid with long axis $2.9 \mu \mathrm{m}$ and short axis $2.4 \mu \mathrm{m}$ (which is reasonable to assume, since the bare particle diameter was determined as approx. $2.4 \mu \mathrm{m}$ and E. coli width is $0.5 \mu \mathrm{m}$ ) yields an apparent Stokes diameter of $2.8 \mu \mathrm{m}$, which compares well with the measured peak value of $2.83 \mu \mathrm{m}$. That is plausible, as the favoured arrangement of the attachment of the particleE. coli complex for $\mathrm{c} 1$ is observed to be along the E. coli length side (as illustrated in Figure 2 and observed for low bacteria concentrations, cp. Fig. $4 \mathrm{~b}$ ).

While single bacteria binding for the lowest concentration c1 permits above explanation in terms of an equivalent diameter, a variety of binding numbers and arrangements can occur for higher concentrations, effectively complicating the use of an analytical model. A further indication for a mixture of binding modes and different number of bacteria per particle is the obvious broadening of the distributions with increasing concentration. However, there is an excellent agreement between peak values and distribution shapes for measurements of the same concentration values, suggesting excellent repeatability of the measurement method. The employed peak fitting furthermore allows us to distinguish between all tested $E$. coli concentrations with the current prototype. The diameter increases in a slightly non-linear fashion with increasing concentration, indicating that at higher concentrations saturation takes place - clearly, at some point all possible binding sites on particles should be occupied by bacteria, so that the ensemble diameter cannot increase any further (for the sake of simplicity not regarding agglomerates, which should constitute only a minor fraction of the species in the sample).

Since the calculated hydrodynamic radius of reference distribution is slightly shifted to the left (i.e., it is in close proximity, but still not precisely $2800 \mathrm{~nm}$ ), additional verification of the feasibility of the tracking system was necessary. The tracking of bare 
Dynabeads ${ }^{\mathrm{TM}}$ M-270 Carboxylic Acid MPs was carried out and the distribution of calculated hydrodynamic diameter can be seen in Figure 11.

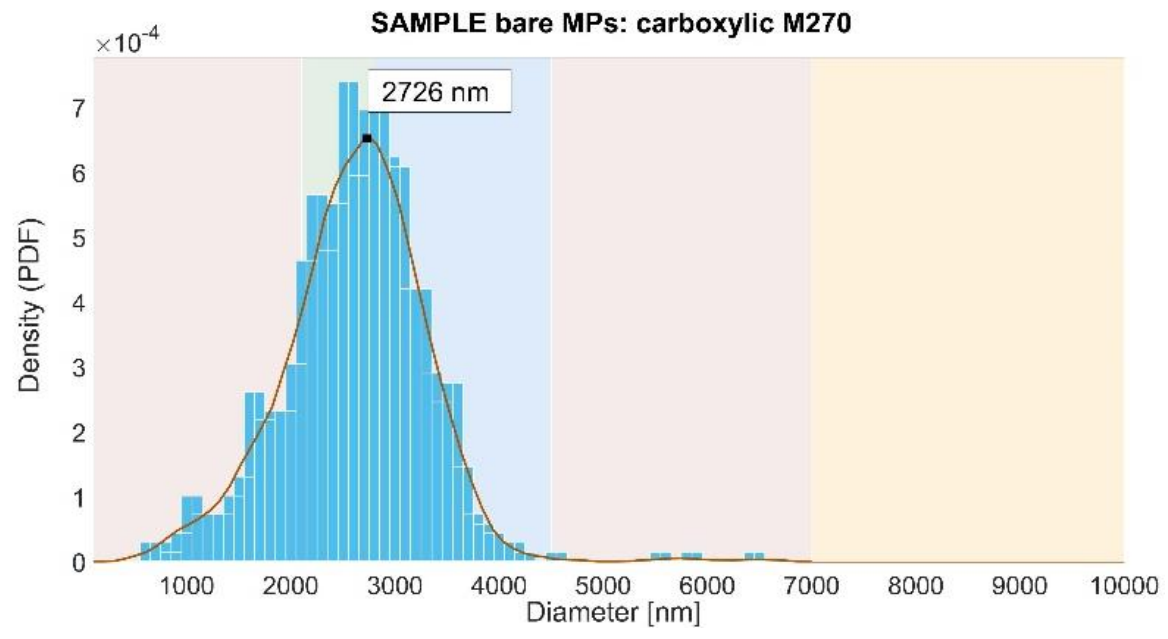

Figure 11. Distribution for calculated hydrodynamic diameter for bare Dynabeads ${ }^{\mathrm{TM}} \mathrm{M}-270$ Carboxylic Acid.

This suggests high accuracy of the system since the claimed $(\sim 2.7 \mu \mathrm{m})$ and the calculated diameter of the particle Dynabeads ${ }^{\mathrm{TM}}$ MP-270 agree within a few percent. Possible explanations for obtaining lower diameter values for Dynabeads ${ }^{\mathrm{TM}}$ MP-280 Streptavidin could be: a) different physical properties resulted in varying molecule interactions, i.e., hydrophobic and hydrophilic nature of M-280 and M-270, respectively, or variability of diffusive double layer (-COOH particles represent a charge, therefore, their diffusive double layer was greater, hence resulting in an apparently bigger measured diameter of M270 particles). This suggestion, together with improved camera settings (fps) could provide more detailed information about thermal fluctuations within the liquid. Dynamic response of bacteria exposed to different external conditions could be examined and $x$ and $y$-displacements could be compared. It is suggested that the association of factors such as temperature and viscosity is investigated in future studies and different buffer solutions are tested. Moreover, the calibration of the systems and validation of the results must be conducted to determine the real ranges corresponding to the specific concentration of bacteria within samples.

Author Contributions: Conceptualization, C.H, G.K. and A.M.; methodology, A.M.; software, A.M., C.H and G.K.; validation, C.H, G.K and I.G.; formal analysis, A.M; investigation, A.M, C.H and G.K.; writing - original draft preparation, A.M and I.G.; writing - review and editing, C.H., G.K and I.G.; supervision, G.K and I.G.; project administration, I.G.; funding acquisition, I.G.

Funding: This research was partially funded by the Austrian Science Fund (FWF) Stand-Alone Project 'Magnetic Microfluidic Biosensor for the Detection and Quantification of Biomolecules' with Project No. P28544-N30. This paper is part of the ANTARES project that has received funding from the European Union's Horizon 2020 research and innovation programme under the European Union's Horizon 2020 research and innovation programme with grant agreement No. 664387.

Conflicts of Interest: The authors declare no conflict of interest.

\section{References}

1. Lazcka, O.; Del Campo, F. J.; Muñoz, F. X. Pathogen detection: A perspective of traditional methods and biosensors. Biosens. Bioelectron. 2007, 22, 1205-1217.

2. Hameed, S.; Xie, L.; Ying, Y. Conventional and emerging detection techniques for pathogenic bacteria in food science: A review. Trends Food Sci. Technol. 2018, 81, 61-73.

3. Heo, J.; Hua, S.Z. An Overview of Recent Strategies in Pathogen Sensing. Sensors 2009, 9, 4483-4502. 
4. Yesilkoy, F.; Terborg, R.; Pello, J. et al. Phase-sensitive plasmonic biosensor using a portable and large field-of-view interferometric microarray imager. Light Sci. Appl. 2018, 7, 17152

5. Pedrero, M.; Campuzano, S.; Pingarrón, J.M. Electroanalytical Sensors and Devices for Multiplexed Detection of Foodborne Pathogen Microorganisms. Sensors 2009, 9, 5503-5520.

6. Gonzalez-Guerrero, A.; Maldonado, J.; Herranz, S.; Lechuga, L. Trends in photonic lab-on-chip interferometric biosensors for point-of-care diagnostics. Anal. Methods, 2016, 8, 8380-8394.

7. Jang, A.; Zou, Z.; Lee, K.K.; Ahn, C.H.; Bishop, P.L. State-of-the-art lab chip sensors for environmental water monitoring. Meas. Sci. Technol. 2011, 22, 032001

8. Giouroudi, I.; Kokkinis, G. Recent Advances in Magnetic Microfluidic Sensors. Nanomaterials 2017, 7, 171.

9. Giouroudi, I.; Keplinger, F. Microfluidic Biosensing Systems using Magnetic Nanoparticles. Int. J. Mol. Sci. 2013, 14, 1853518556.

10. Sackmann, E.K.; Fulton, A.L.; Beebe, D.J. The present and future role of microfluidics in biomedical research. Nature 2014, 507, 181-189.

11. Mairhofer, J.; Roppert, K.; Ertl, P. Microfluidic Systems for Pathogen Sensing: A Review. Sensors 2009, 9, 4804-4823.

12. Reshes, G.; Vanounou, S.; Itzhak Fishov, I.; Feingold, M. Cell Shape Dynamics in Escherichia Coli. Biophys. J. 2008, 94, 251-64.

13. Malec, A.; Kokkinis, G.; Haiden, C.; Giouroudi, I. Biosensing System for Concentration Quantification of Magnetically Labeled E. Coli in Water Samples. Sensors 2018, 18, 2250.

14. Kokkinis, G.; Plochberger, B.; Cardoso, S.; Keplinger, F.; Giouroudi, I. Microfluidic, dual purpose sensor for in-vitro detection of Enterobacteriaceae and biotinylated antibodies. Lab Chip 2016, 16, 1261-1271.

15. Jamalieh, M.; Kokkinis, G.; Haiden, C.; Berris, T.; Keplinger, F.; Giouroudi, I. Microfluidic Platform for Pathogen Load Monitoring. Microelectron. Eng. 2016, 158, 91-94.

16. Einstein, A. Investigations on the Theory of the Brownian Movement. Ann. Phys. 1905, 17, 549.

17. Haiden, C. et al. Sizing of Metallic Nanoparticles Confined to a Microfluidic Film Applying Dark-Field Particle Tracking. Langmuir 2014, 30, 9607-9615.

18. Haiden, C. et al. Concurrent Particle Diffusion and Sedimentation Measurements Using Two-Dimensional Tracking in a Vertical Sample Arrangement. Appl. Phys. Lett. 2016 108, 094101.

19. Haiden, C. et al. A Microfluidic Chip and Dark-Field Imaging System for Size Measurement of Metal Wear Particles in Oil. IEEE Sens. J. 2016, 16, 1182-1189.

20. Michalet, X. Mean square displacement analysis of single-particle trajectories with localization error: Brownian motion in an isotropic medium. Phys. Rev. E 2010, 82, 041914

21. Dunderdale, G.; Ebbens, S.; Fairclough, P.; Howse, J. Importance of Particle Tracking and Calculating the Mean-Squared Displacement in Distinguishing Nanopropulsion from Other Processes. Langmuir 2012, 28, 10 997-11 006

22. Hasinoff, S. W. Saturation (Imaging). In Computer Vision, Katsushi I.: Springer US, 2014; pp. 699-701.

23. Crocker, J. C.; Grier, D.G. Methods of Digital Video Microscopy for Colloidal Studies. J. Coll. Int. Sci. 1996, 179, $298-310$. 